\title{
Antiimperialismo latinoamericanista en la generación Jel Bicentenario
}

\section{Latin Americanist anti-imperialism in the generation of the Bicentennial}

\section{Anti-imperialismo Latino-americano na geração do Bicentenário}

\author{
Andrés Mora-Ramírez \\ Académico, Centro de Investigación y Docencia en Educación. \\ Universidad Nacional de Costa Rica \\ Recibido: 3/3/2015 Aceptado: 6/5/2015 \\ http://dx.doi.org/10.15359/tdna.31-58.6
}

\section{Resumen}

En este artículo sostengo la tesis de que uno de los componentes centrales del llamado cambio de época en América Latina, en el siglo XXI, corresponde a una vertiente antiimperialista que se expresa con claridad en dos dimensiones: el acento bolivariano-martiano y antiimperialista del discurso político de los exponentes de la generación del Bicentenario; y segundo, su concreción en la nueva arquitectura de la integración regional, de signo posneoliberal. Se argumenta

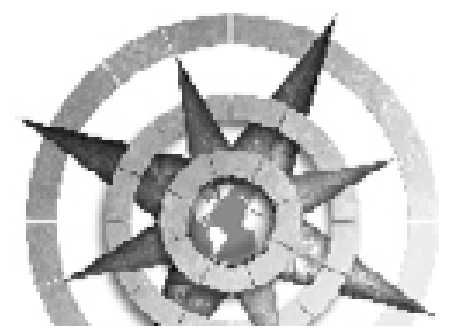

que el proceso revolucionario, antiimperialista y nacional-popular latinoamericano del siglo XXI, fortaleció la autonomía de la región y su posición en el sistema internacional, desligando su política exterior del sometimiento tradicional a los Estados Unidos.

Palabras clave: Antiimperialismo latinoamericano, pensamiento latinoamericano, integración regional latinoamericana, procesos revolucionarios, posneoliberalismo

\section{Abstract}

In this article we argue that one of the central components of the so-called change of era in Latin America in the 21st. century, corresponds to an anti-imperialist aspect that is clearly expressed in two dimensions: the 
Bolivarian-Martian and anti-imperialist tone of the speech of the political exponents of the Generation of the Bicentennial; and second, its concretion in the new architecture of regional integration, of post-neoliberal sign. It is argued that the Latin American revolutionary, anti-imperialist and national-popular process of the 21st. century, strengthened the autonomy of the region and its position in the international system by decoupling the traditional foreign policy submission to the United States.

Keywords: Latin American Antiimperialism, Latin American Thinking, Latin American Regional Integration, Revolutionary processes, post-neoliberalism

\section{Resumo}

Neste artigo, argumenta-se que um dos componentes centrais da chamada mudança de época na América Latina no século XXI, corresponde a um aspecto anti-imperialista que está claramente expresso em duas dimensões: o acento da Bolivar-Marti e anti-imperialista do discurso político da geração do Bicentenário; e em segundo lugar, a sua realização na nova arquitetura de integração regional, do signo pós-neoliberal. Argumenta-se que o processo revolucionário, antiimperialista e nacional-popular latinoamericano do século XXI, reforçou a autonomia da região e sua posição no sistema internacional, dissociando sua política externa da tradicional submissão a-os Estados Unidos.

Palavras chave: Anti-imperialismo latinoamericano, pensamento latinoamericano, integração regional latinoamericana, processo revolucionário

No aconsejemos la diplomacia. Nuestra defensa está en la cultura, en realizar efectivamente una función de cultura. Hacer pensar al país (...). Cuando seamos grandes por nuestra cultura, ique vengan los Estados Unidos!, que entonces sólo recibiremos de ellos lo que sea grande también (Gamboa, 1971, pp. 214-216).

Omar Dengo, discurso en la Universidad Popular, 1926.

En su obra clásica Imperialismo y liberación. Una introducción a la historia contemporánea de América Latina, el intelectual mexicano Pablo González Casanova (1991, p. 50) afirma que la historia de las masas populares y de sus luchas, en cualquier lugar del mundo,

es mucho más rica y más compleja que la de las clases gobernantes. Encierra más combinaciones posibles, más recursos inesperados...

En nuestra región, desde finales de los años 1990 y en lo que transcurre del siglo XXI, asistimos a un conjunto de 
procesos de ese tipo: ricos y complejos en sus combinaciones posibles, en sus giros emancipadores y sus recursos inesperados, y en definitiva, en las transformaciones que propician sus actores, con implicaciones de diversa magnitud para los Estados nacionales y sus sociedades, en el orden de lo político, lo socioeconómico, lo cultural y lo ideológico.

El ascenso y protagonismo de nuevos movimientos sociales, herederos de una tradición de lucha forjada en tiempos de dictaduras militares, puso en tensión y en no pocos casos fracturó la hegemonía neoliberal, lo que abrió el camino a la configuración de un amplio arco de proyectos políticos de fuerte base nacional-popular, que accedieron sucesivamente al poder en varios países, con triunfos inobjetables alcanzados en elecciones libres. Nuevos liderazgos políticos surgidos de los sectores tradicionalmente oprimidos, obreros, campesinos, pueblos originarios, antiguos movimientos guerrilleros, de la academia, de la iglesia católica identificada con la teología de la liberación, e inclusive de sectores nacionalistas de las fuerzas armadas, han estado al frente de dichos procesos y su impronta ha llevado a varios autores a caracterizarlos como la generación del Bicentenario.
Con acierto, estas transformaciones han sido definidas como un cambio de época en América Latina, y en registros mucho más románticos del discurso, se le ha llamado también el fin de la larga noche neoliberal. En esta ponencia, se sostiene la tesis de que uno de los componentes centrales de ese giro epocal, múltiple y diverso, corresponde a una vertiente antiimperialista que recorre transversalmente las luchas, debates y creaciones de esta primera década y media del siglo XXI, y que se expresa con claridad en dos dimensiones, mutuamente entrelazadas, que se analizarán en este trabajo: la fuerte raíz o acento bolivariano-martiano y antiimperialista del discurso político nuestroamericano de los exponentes de la generación del Bicentenario; y su correlato en la nueva arquitectura de la integración regional, de signo posneoliberal, en tanto intenta revertir el impacto negativo del neoliberalismo impuesto en América Latina desde los años 1970, y articulada en oposición al proyecto panamericano del imperialismo estadounidense.

\section{Sobre el imperialismo estadouni- dense y el antiimperialismo latinoa- mericano: ayer y hoy}

En 1894, José Martí publicó en Nueva York un artículo titulado La verdad sobre los Estados Unidos, con 
el que inauguró una nueva sección en el diario independentista cubano Patria, expresamente destinada a divulgar los sucesos que

demuestran las dos verdades útiles a nuestra América: el carácter crudo, desigual y decadente de los Estados Unidos, y la existencia, en ellos continua, de todas las violencias, discordias, inmoralidades y desórdenes de que se culpa a los pueblos hispanoamericanos (En Hart, 2000: p. 233).

La época en que Martí escribe este texto es también la del surgimiento de figuras y procesos determinantes en la configuración del imperialismo estadounidense: John D. Rockefeller consolidaba el primer trust moderno, la Standard Oil Company; el almirante Alfred Mahan desarrollaba su teoría de las invasiones estratégicas, colocando a la armada y los marines como bastiones del dominio militar; y James Blaine promovía el panamericanismo como doctrina de dominio comercial y diplomático, a través de la primera Conferencia Internacional Americana (1889-1891), (González, 1991). Esto por citar solo tres ejemplos de personajes cuya impronta quedó marcada --casi literalmente-- a sangre y fuego en el diseño y evolución de la política exterior interamericana de los Estados Unidos. En ese escenario, la obra intelectual y política de Martí dio el santo y seña de lo que, desde entonces, se consolidaría como una tradición política y cultural de primer orden en la construcción de la identidad -múltiple y diversa- de nuestra América: el antiimperialismo latinoamericanista.

Este antiimperialismo latinoamericanista, que hoy reconocemos como rasgo identitario de actores políticos y sujetos sociales en distintas latitudes de nuestra América, prolonga un legado intelectual construido durante varias generaciones -que entreteje, por ejemplo, a Martí con Sandino-, y que tiene como momento inaugural la resistencia cultural, en su amplio sentido, que se enarboló frente al expansionismo estadounidense de finales del siglo XIX y el devenir de su desarrollo imperialista. Se trata, pues, de una suerte de nacionalismo latinoamericano, que encuentra sustento fundacional en "la historia propia" como pilar de contención frente a "las intervenciones foráneas", y en la aspiración de formar "una comunidad de naciones que originaran la fuerza necesaria para detener al imperialismo" (Cuevas, 2008, pp. 26-27).

No por casualidad, la coyuntura política y las tensiones ideológicas de los últimos quince años en la región nos remiten a esa forma particular del antiimperialismo, y a la recomendación 
martiana de permanente estudio y vigilancia sobre los Estados Unidos. Esto es especialmente cierto ahora, porque una de las constantes del discurso político del presidente Barack Obama y las autoridades en Washington ha sido la necesidad -casi la urgencia-- de que su país recupere el liderazgo hemisférico y mundial. Y resulta evidente que reconstruir esa hegemonía perdida ante la reconfiguración del mundo multipolar, implica tensiones y conflictos con una América Latina que ha empezado a transitar nuevos caminos, cada vez más alejados de la égida de la potencia del norte.

Desde esta perspectiva, es posible ubicar las agresivas políticas republicanas de las dos administraciones del exmandatario George W. Bush (2001-2009), y las políticas del smart power de los dos gobiernos de Obama (desde 2009 a la fecha), en el marco más amplio del proyecto de expansión imperialista estadounidense: ese que inicia en 1847-1848 en la guerra contra México (aunque algunos autores lo señalan en 1823, con el nacimiento de la Doctrina Monroe) (Quesada, 2001, p. 28), que luego adopta rasgos monopólico-capitalistas a partir de 1880 y que, desde 1898-1902, con la primera guerra imperialista que desembocó en la anexión de Cuba, Puerto Rico,
Filipinas, Hawai y Guam, define su perfil intervencionista (en lo político, militar y cultural) dominante a lo largo del siglo XX.

En el siglo XXI, la persistencia de las agresiones contra Cuba -solo maquilladas por la reciente distensión diplomática--, o las maniobras golpistas y de desestabilización en Venezuela, Bolivia y Ecuador, lo mismo que en Honduras, Paraguay, $y$ recientemente en Argentina y Brasil, adquieren una profundidad político-cultural mayor a la luz de esa mirada histórica sobre el comportamiento y las reformulaciones contemporáneas del imperialismo en América Latina. Lo que hoy observamos, entonces, no es otra cosa sino el modus operandi de la principal potencia militar y, en el fondo, el comportamiento propio del imperialismo permanente: con este concepto, el historiador Rodrigo Quesada define el estadio del desarrollo y expansión del sistema capitalista mundial que madura a partir de finales del siglo XIX, y en concreto, desde el año 1898, con la guerra hispano-antillano-norteamericana: la primera guerra inter-imperialista. El imperialismo permanente, explica Quesada: No solo supone una forma inédita de acumulación a escala mundial, apuntalada por la compañía trasnacional que funciona 
como pivote sobre el que se apoya también la supuesta globalización, sino también (...) el expansionismo norteamericano brutal y desalmado con todas las implicaciones que ello tiene. $(2012$, p. 40)

Tal expansionismo ha sido posible, fundamentalmente, por la hegemonía militar, que abre el camino a la apropiación de recursos y mercados, leit motiv de ese largo historial de intervenciones abiertas y solapadas.

Ahora bien, si, como sostiene el historiador costarricense, la guerra de 1898 en Cuba, Puerto Rico y Filipinas fue el escenario para que se pusieran en práctica "usos militares desconocidos hasta entonces (apenas insinuados en la India durante la dominación británica), además del genocidio y una relación sumamente estrecha entre la prensa, la industria militar y el gobierno norteamericano" (Quesada, 2012, p. 41); más de un siglo después, las intervenciones en marcha en el mundo árabe y en otras regiones del mundo, apoyadas de nuevo por la poderosa entente mediática, económica y política, tan solo reafirman la dominación y la locura autodestructiva del imperialismo permanente, frente al cual hoy deben plantarse todas las banderas de lucha, resistencia y de humanidad.
En ese sentido, el legado de Martí -el que expuso en sus escritos y el que templó con su sangre y su propia vida- constituye un arsenal de ideas y lecciones políticas que no pierden vigencia. En el ensayo Nuestra América de 1891, por ejemplo, nos advierte que:

Sobre algunas repúblicas está durmiendo el pulpo. Otras, por la ley del equilibrio, se echan a pie a la mar, a recobrar, con prisa loca, los siglos perdidos. (...) Pero otro peligro corre, acaso, nuestra América, que no le viene de sí (...). El desdén del vecino formidable [los Estados Unidos], que no la conoce, es el peligro mayor de nuestra América (En Hart, 2000, pp. 210-211).

Como contrapunto necesario a ese desdén, que incuba los peores vicios humanos y desata los apetitos imperiales, Martí creía que no había otra alternativa sino la marcha unida de los pueblos latinoamericanos, "en cuadro apretado, como la plata en las raíces de los Andes" (p. 220). Una exhortación que comprendieron, como nunca antes, los representantes de la generación del Bicentenario. 
La generación del Bicentenario: la vuelta de lo nuestroamericano a la lucha antiimperialista.

En diciembre del año 2006, en el marco de la II Cumbre Social por la Integración de los Pueblos realizada en Bolivia, el presidente de Ecuador, Rafael Correa, dirigió un mensaje a los participantes de este foro en el que, echando mano del lenguaje metafórico, propuso una sugestiva interpretación de los tiempos que empezaba a vivir nuestra América por esos años, y sostuvo que "la región no está viviendo una época de cambios, está viviendo verdaderamente un cambio de época. La larga y triste noche neoliberal finalmente está siendo derrotada y un nuevo amanecer se avizora en cada rincón de América Latina" (Minga Informativa, 9 de diciembre de 2006).

Con gobiernos progresistas y nacional-populares sembrados en toda la región, gracias a los triunfos alcanzados en elecciones democráticas incuestionables; con proyectos de una nueva integración regional en ciernes, como la Alianza Bolivariana para los Pueblos de Nuestra América (ALBA), la Unión de Nacionales Suramericanas (UNASUR) o la Comunidad Estados Latinoamericanos y del Caribe (CELAC); con movimientos sociales comprometidos con los procesos constituyentes en Bolivia y Ecuador; con un pueblo venezolano que maduraba en la lucha abierta contra el fascismo imperialista y en la solidaridad internacionalista; y con liderazgos indiscutibles en las figuras de los presidentes Hugo Chávez, Néstor Kirchner (ambas, notables ausencias en el actual panorama latinoamericano), Lula da Silva, Evo Morales y el propio Correa, y más tarde Manuel Zelaya, Daniel Ortega, Fernando Lugo, Cristina Fernández, Michelle Bachelet, Dilma Rousseff o Nicolás Maduro, la región efectivamente inició su tránsito por una época inédita: después de décadas de opresión, por fin los pueblos daban el paso de la resistencia a la construcción de alternativas.

Fue en ese contexto que el periodista argentino Luis Bruschtein acuñó el concepto de generación del Bicentenario, precisamente para referirse al colectivo de presidentes y presidentas que alcanzaron el poder por la vía electoral en los últimos 15 años, y cuyo grado de afinidad y sintonía para avanzar en propuestas de concertación y unidad políticas, no registra parangón en la historia contemporánea del continente. Para Bruschtein:

Se podrá estar de acuerdo o no con ellos, pero no existe otro momento en la historia de América 
Latina en que un grupo de presidentes haya estrechado ese nivel de contactos, afinidades y confianza mutua y seguramente marcarán, como generación, esta etapa de la historia del subcontinente. Serán odiados por unos y amados por otros, pero seguramente recordados como la generación del Bicentenario. (24 de abril, 2010: s/p).

En nuestra perspectiva, un elemento central que ha permitido este estrechamiento de vínculos y voluntades, tanto en el discurso como en la praxis política, es la vocación nuestroamericana de esta generación, lo que responde no solo a una cuestión coyuntural sino, en buena medida, a la memoria profunda de la violencia imperialista sufrida por nuestros pueblos y al temple telúrico -forjado en numerosos combates-- de las organizaciones, movimientos y partidos que construyeron las condiciones para que fuese posible la llegada al poder de los nuevos presidentes y presidentas. Rastrear este hilo conductor que entreteje la vocación unionista a través de los siglos y hasta nuestros días resulta fundamental para comprender las herencias, las aspiraciones y utopías compartidas entre aquella generación de libertadores, los precursores del antiimperialismo de los siglos XIX y XX, y la generación del Bicentenario.
En este sentido, la idea de la unidad posible de América Latina debe ser comprendida como uno de los ejes articuladores de la construcción de nuestras identidades culturales y de las luchas de liberación de los pueblos latinoamericanos, tanto en sus triunfos como en sus derrotas, en un itinerario liberador que nos lleva de Simón Bolívar, quien en el primer cuarto del siglo XIX delinea el futuro posible del continente, que se sacude las cadenas del imperio español, a partir de la unión y la "idea grandiosa" de "formar de todo el Mundo Nuevo una sola nación con un solo vinculo que ligue sus partes entre sí y con el todo (...) en alguna época dichosa de nuestra regeneración" (Bolívar, 1985, p. 61); también nos remite al propio Martí que, hacia fines de esa centuria, despliega sus mejores armas intelectuales para impulsar la tesis -complementaria de la de Bolívar- de la unidad necesaria sustentada bajo el signo triple del latinoamericanismo, el humanismo y el antiimperialismo.

Con Martí, la idea de la unidad nuestroamericana, que parte como respuesta original al problema del colonialismo español, alcanza su madurez en el conjunto del pensamiento latinoamericano. A la vista del gigante que se levantaba en el Norte, y en el marco del Congreso 
de Washington de 1889, primer antecedente del panamericanismo, el prócer cubano -testigo de excepción de aquella cita- alerta sobre la necesidad de declarar la segunda independencia de los pueblos de nuestra América, como respuesta "unánime y viril" ante los Estados Unidos y la "política secular y confesa de predominio de un vecino pujante y ambicioso, que no los ha querido fomentar" (Martí, 2005, pp. 56-57).

Esta doble raíz bolivariana y martiana que constituye el discurso nuestroamericano también se expresó con fuerza en el siglo XX: en un mundo que se enfilaba ya a la colisión entre el capitalismo y socialismo real, Augusto César Sandino, enfrentado al imperialismo estadounidense que ocupaba militarmente a Nicaragua, y "que ya no atiende a teorías de derecho y de justicia pasando sin respeto alguno por sobre los inconmovibles principios de independencia de las fracciones de la NACIONALIDAD LATINOAMERICANA" (Sandino, s.f.), propuso a los pueblos latinoamericanos -en 1929. formar una alianza o federación de los países con sus propias instituciones políticas, jurídicas, militares, financieras, de transporte y comunicaciones. Y en 1962, ante la acción concertada de los Estados Unidos y casi la totalidad de los gobiernos cómplices de América Latina para expulsar a Cuba de la Organización de Estados Americanos (OEA), y con el bloqueo económico ya en marcha, el líder cubano Fidel Castro proclamó en la Plaza de la Revolución la Segunda Declaración de La Habana, manifiesto clave en el que se entiende el antiimperialismo como un empeño de hermandad y solidaridad entre pueblos "que padecen las mismas miserias, albergan los mismos sentimientos, tienen el mismo enemigo, sueñan todos con un mismo mejor destino, y cuentan con la solidaridad de todos los hombres y mujeres honrados del mundo" (Castro, 2009, pp. 73-74).

En la terrible década perdida del neoliberalismo, los años noventa, dos voces que provienen de trayectorias distintas se encuentran para perfilar lo que entendemos como el punto de partida, en términos de la toma de conciencia sobre sus responsabilidades históricas, de la generación del Bicentenario: en 1993, en el marco de la reunión del Foro de Sao Paulo en La Habana, Fidel Castro planteó la necesidad de "crear una esperanza para el futuro" de los pueblos, a partir de la unidad nuestroamericana, de la integración política y económica como "una cuestión de supervivencia", en un mundo "de grandes gigantes económicos e industriales, de 
grandes comunidades económicas y políticas". Y preguntaba: "¿Qué menos podemos hacer nosotros y qué menos puede hacer la izquierda de América Latina, que crear una conciencia a favor de la unidad?" (Castro, 2009, pp. 235-236).

Un año después, en diciembre de 1994, un joven y rebelde líder venezolano, Hugo Chávez, dio un discurso en el Aula Magna de la Universidad de La Habana, cuyo contenido y visión estratégica debería estudiarse con mayor interés en nuestras academias y organizaciones políticas, toda vez que en esa intervención perfiló el programa ideológico de lo que, años después, se convirtió en la Revolución Bolivariana; y además, anunció el proyecto de integración continental que sentó las bases de la actual CELAC: "un proyecto estratégico continental de largo plazo", que en un horizonte de veinte a cuarenta años, permitiera el desarrollo de un modelo económico y político alternativo, soberano y complementario para la región. "Una asociación de Estados latinoamericanos (...) que fue el sueño original de nuestros libertadores (...). Un congreso o una liga permanente donde discutiríamos los latinoamericanos sobre nuestra tragedia y sobre nuestro destino"; que hiciera del siglo XXI "el siglo de la esperanza y de la resurrección del sueño bolivariano, del sueño de Martî" (Chávez, 1994).

Chávez, por entonces un teniente coronel de paracaidistas del ejército venezolano recién indultado, visitaba Cuba por primera vez, en lo que sería el inicio de una relación tan profunda e innovadora en sus alcances unionistas, como no se registraba en las últimas décadas entre dos naciones latinoamericanas. Según se recuerda, el líder bolivariano arribó el 14 de diciembre a La Habana en un vuelo comercial y, para su sorpresa, fue recibido en el Aeropuerto Internacional José Martí por el propio Fidel Castro. El historiador cubano Eusebio Leal dijo de aquel encuentro:

Fidel descubrió en Chávez a un diamante que alcanzaría las cotas más altas en el discurso político, revolucionario e internacionalista. Creo que el que lo vio todo con claridad, nitidez y visión, que alcanza el tiempo futuro, fue Fidel, quien cobró una simpatía infinita por él (Correo del Orinoco, 13 de marzo de 2013).

En 2003, en un acontecimiento rico en significados de vocación latinoamericanista, y que ampliaba el círculo de representantes de la generación del Bicentenario, los presidentes Fidel Castro, Hugo Chávez y Lula da Silva -el trabajador metalúrgico que 
recién había ganado la presidencia de Brasil-- destacaron como invitados de honor a la toma de posesión de Néstor Kirchner, en la Casa Rosada de Buenos Aires: precisamente allí donde no hacía mucho tiempo se paseaba una galería de mandatarios y tecnócratas neoliberales funcionales al imperialismo estadounidense. Castro afirmó en aquella ocasión, ante un público masivo que lo escuchó en la Facultad de Derecho de la Universidad de Buenos Aires: "El sol que vi esta mañana en el homenaje a Martí y a San Martín, el sol que vi al llegar a este país y el que siento en esta escalinata. Este sol es el de las ideas que pueden traer paz, que pueden traer soluciones" (Bruschtein, 27 de mayo de 2003).

Un año más tarde, en 2004, la firma del acuerdo de creación del ALBA entre los gobiernos de Cuba y Venezuela anunciaba la gestación de una perspectiva de la integración radicalmente distinta a los enfoques panamericanistas dominantes en las últimas décadas; además, el hecho tuvo una connotación mayor: representó una suerte de relevo generacional en las luchas antiimperialistas y latinoamericanistas en el continente, al pasar la estafeta de la figura señera de Castro al presidente Chávez, quien venía de vencer, gracias a la acción del pueblo y los militares patriotas venezolanos, el golpe de Estado y las maniobras imperialistas para sacarlo de Miraflores y acabar con su vida.

En 2005, en la Cumbre de las Américas de Mar del Plata, cuando pueblos y líderes latinoamericanos enterraron el proyecto panamericano del Área de Libre Comercio de las Américas, ALCA, el gran tratado comercial con el que soñaban el expresidente estadounidense Bush y los neoliberales criollos, Néstor Kirchner (2005) dijo a los mandatarios de todo el continente que ya era tiempo de "construir un sistema que nos vuelva a contener a todos en un marco de igualdad y nos vuelva a devolver la esperanza y la posibilidad de construir obviamente un mundo distinto y una región que esté a la altura de las circunstancias". Ese orden distinto al que apelaba Kirchner cristalizó en 2011 con la creación de la CELAC, el primer organismo continental del que no participan Estados Unidos y Canadá: precisamente, quince años después de aquellas palabras de Chávez en la Universidad de La Habana.

En esta clave de construcción discursiva, y desde la mirada de los actores y sujetos sociales de la generación bicentenaria, en la CELAC convergen sueños y proyectos que 
entienden que la idea de la unidad y la integración nuestroamericana todavía puede ser un faro de esperanza en la larga batalla por la liberación de América Latina, para poner fin a los anclajes impuestos históricamente por los Estados Unidos para perpetuar su dominación mediante la ideología del panamericanismo, como eje rector de la política exterior, y de un sistema jurídico-político afín a ese propósito, y que se articuló en torno a la OEA.

\section{Integrarse para vencer: del ALBA a la CELAC}

A inicios del siglo XXI en nuestra América, nuevamente hemos conocido empeños originales y audaces a favor de la unidad y la integración, como las ya mencionadas ALBA, UNASUR y CELAC, que emergen en un contexto regional marcado por las luchas antineoliberales, el ascenso de lo nacional-popular y un giro político progresista que se expresa con mayor o menor intensidad en cada país.

Esta nueva integración tiene tres rasgos fundamentales: el primero, su carácter posneoliberal, porque surge en el contexto de lo que Ana Esther Ceceña (2008) llama un posneoliberalismo nacional alternativo, a saber, el conjunto de políticas de superación del paradigma neoliberal y de creación del poder participativo que se impulsan, sobre todo, desde Venezuela, Bolivia y Ecuador. El segundo rasgo es su carácter independiente y antiimperialista, porque se construye sobre la base de procesos de recuperación de la soberanía popular, nacional, energética, territorial. Así, se desmarca de la influencia y el poder que ejercieron los organismos internacionales y los EE.UU. hasta finales del siglo XX (Regueiro, 2008). $Y$ finalmente, es multidimensional (Suárez, 2008) porque, además de lo económico, incorpora aspectos esenciales de la vida social como la cultura, la educación, el deporte, la salud, la comunicación social y los derechos humanos en un sentido amplio.

En este marco, impulsada por los sectores populares y nacionalistas latinoamericanos, e inspirada en el pensamiento martiano-bolivariano, el ALBA es la iniciativa que más lejos ha llevado las posibilidades de ensayar alternativas al capitalismo y que procura forjar "el equilibrio del mundo" -al decir de Martí-, tarea que exige, primero, subvertir y transformar el orden económico y social existente. El ALBA representa hoy la avanzada de la unidad e integración latinoamericana que, al desafiar la lógica ortodoxa del capitalismo neoliberal, 
señala un camino posible para la sobrevivencia de la especie humana.

El Manifiesto Bicentenario de Caracas, consolidando la Nueva Independencia que se aprobó en la cumbre extraordinaria del ALBA, celebrada en Caracas el 19 de abril de 2010, entre muchos apartados importantes, resalta precisamente la manera en que este espacio de integración "se ha convertido en un elemento cohesionador y dinamizador para avanzar, en las actuales circunstancias históricas, hacia el objetivo superior que constituye el Sueño Bolivariano de crear la más grande República que haya existido y la unidad regional", y como alternativa al imperialismo y al "capitalismo globalizado que hoy amenaza la existencia de la Madre Tierra y la supervivencia de la humanidad" (ALBA-TCP, 2010).

Por otra parte, como contrapunto indispensable a la OEA, ese tradicional brazo político de proyección de los intereses estadounidenses en el continente, el nacimiento de la CELAC se inscribe en la disputa entre panamericanismo y latinoamericanismo. Es decir, entre, por un lado, el imperialismo comercial, cultural y militar estadounidense, que desde el último cuarto del siglo XIX se expande hacia el sur de su frontera; y por el otro, el empeño bicentenario de asociación y de unión en una comunidad de pueblos y estados realmente nuestra. La posibilidad de una gran unión latinoamericana sin la tutela de los Estados Unidos alarma a los imperialistas, de ahí que no sean pocas las presiones que ya reciben los gobiernos de parte de Washington, para desvirtuar y descarrilar la al nuevo organismo.

La CELAC aparece en el horizonte latinoamericano en momentos en que, en el nivel global, se va configurando un nuevo orden multipolar, en medio de las ruinas del viejo orden de la segunda posguerra del siglo $\mathrm{XX}$ : ese que la crisis civilizatoria del capitalismo y las locuras bélicas de las potencias occidentales van destrozando poco a poco. Para que se convierta, efectivamente, en el gran aporte de la generación del Bicentenario al siglo XXI, esta iniciativa deberá enfrentar en lo inmediato dos grandes desafíos: el primero, consolidarse como una organización de profundo carácter latinoamericanista, es decir, que discuta y analice nuestros problemas y sus posibles soluciones con una perspectiva desde acá; y que su proceder sea consecuente con la democracia que, desde diversas vías y experiencias, vienen buscando y forjando los pueblos latinoamericanos. 
El segundo desafío de la CELAC será desmontar el andamiaje político, jurídico e institucional de la OEA, y sustituirlo por un sistema cualitativamente distinto y superior, democrático, participativo, pacífico y humanista, que supere de una vez por todas el esquema hegemonista impuesto por los Estados Unidos y sus aliados nacionales. Y esto implica conciliar los intereses de gobiernos con marcadas diferencias ideológicas, dentro del objetivo mayor de la unidad regional.

La nueva visión de la integración latinoamericana ha sido el campo de disputa ideal para que la generación del Bicentenario emprendiera, una vez más, la batalla cultural por la unión latinoamericana y caribeña, apoyada en la emancipación social, popular y antineoliberal, refrendada ahora por los presagios de colapso del sistema mercadocéntrico y especulativo que rige los destinos de los países industrializados, y que en América Latina fue vanguardia de la neocolonización impulsada desde los años 1990. La nueva Comunidad de Estados podría darle a la humanidad una esperanza más en el camino de alcanzar esa utopía de paz, soberanía, autodeterminación y bienestar de las grandes mayorías, lo que Martí definió de modo brillante como el equilibrio del mundo (en Hart, 2000, p. 240). Es decir, un nuevo orden social y de las relaciones internacionales, como aún no lo ha conocido la civilización moderna.

Ese ideario martiano ya está presente en los principios fundadores de la CELAC, y en la Declaración de Caracas del 2011 se expresan en el reconocimiento de la diversidad cultural e identitaria de América Latina y el Caribe, así como "el derecho que tiene cada nación de construir en paz y libremente su propio sistema político y económico", y el respeto a la autodeterminación, la soberanía, la integridad territorial, la no injerencia en los asuntos internos de cada país, y "la protección y promoción de todos los derechos humanos y de la democracia" (CELAC, 2011). No es casual que más de un siglo después, Martí y sus ideas conserven su vigencia. Las amenazas y desafíos siguen siendo, en buena medida, aquellos que conoció y avizoró el prócer cubano: el dilema -ahora renovado- entre el unionismo latinoamericano y el neopanamericanismo imperial, y la tarea pendiente de completar el desarrollo económico, social, cultural y ambientalmente sostenible de nuestras "repúblicas dolorosas de América" (en Hart, 2000, p. 204).

124 Antiimperialismo latinoamericanista en la generación del 


\section{Reflexiones finales}

En este siglo XXI, el proceso revolucionario, antiimperialista y nacional-popular latinoamericano, articulado en torno al eje La Habana-Caracas-Brasilia-Buenos Aires, fortaleció la autonomía política, impulsó la construcción de alternativas de integración y complementariedad en diversos campos (economía y finanzas, infraestructura, seguridad, cultura, educación), así como consensos y horizontes de futuro compartido, como nunca antes había conocido el continente. Gracias a la generación del Bicentenario, nuestra América fortaleció su posición en el sistema internacional, desligando su política exterior del sometimiento tradicional a los Estados Unidos, y al mismo tiempo, abrió nuevo espacios de encuentro en la lógica Sur-Sur (por ejemplo, con África y las potencias emergentes del grupo BRIC: Brasil, Rusia, India y China).

Hoy, sin embargo, una mirada crítica pero al mismo tiempo identificada con los procesos políticos nuestroamericanos, nos arroja un panorama que requiere nuevas interpretaciones y respuestas: a saber, la imagen del repliegue de aquella ebullición emancipadora que desató la rebelión contra el entreguismo neoliberal, y de una pérdida relativa de la iniciativa estratégica por parte de los gobiernos nacional-populares y de los movimientos sociales que, de victoria en victoria, en las urnas y las calles, fueron abriendo un horizonte de posibilidades y de acción en el que encontraron cabida diversos sueños y utopías.

Una lectura de conjunto de la actual correlación de fuerzas revela que el llamado cambio de época en América Latina empieza a conocer sus fronteras, sus límites dentro de la misma situación que ayudó a construir, por lo que se impone un nuevo esfuerzo de creación desde abajo, revolucionario en todo sentido, para convertir los retos e insatisfacciones del presente, en oportunidades que impulsen hacia el futuro las conquistas de la última década y media. Y eso supone, necesariamente, que los gobiernos y los dirigentes recuperen la capacidad de ser voceros e instrumentos para la concreción de la voluntad popular emancipadora, y no su freno. Frente al dilema de estancarse para perecer, o acelerar el paso hacia las transformaciones posneoliberales y poscapitalistas --y por lo tanto, antiimperialistas-- que demanda nuestro tiempo, la única certeza posible es la del protagonismo de los pueblos para romper los nudos de la conformidad, de la inercia, de los intereses de clase y todas aquellas 
cadenas que impiden avanzar hacia la tan anhelada segunda independencia de nuestra América. Y esto no puede hacerse desde otro cause ético, político y cultural que no sea el de la opción por los oprimidos y "los pobres de la tierra" (Martí, 1999, p. 119); y sin perder de vista que la potencia del Norte, dados sus apetitos y su manera de conducir las relaciones con nuestra América, no reconoce ni respeta las normas del Derecho Internacional.

Hoy más que nunca el legado de Martí, pensamiento y acción, mantiene su vigencia como esa "luz de permanente aviso" (en Hart, 2000, p. 241) que sale de su tumba --según nos dejó dicho--, y también de las de cada hombre y mujer que han luchado por la dignidad, la libertad y la independencia aún inconclusa de nuestra América.

\section{Referencias bibliográficas}

ALBA-TCP. (2010). Manifiesto Bicentenario de Caracas, consolidando la Nueva Independencia. Adital. Recuperado de: http://site.adital.com.br/site/ noticia.php?lang=ES\& $\operatorname{cod}=47135$.

Bolívar, S. (1985). Doctrina del libertador (Manuel Pérez Vila, comp.). Caracas: Fundación Biblioteca Ayacucho.

Bruschtein, L. (27 de mayo de 2003). Un mensaje a los que quieren bombardear Cuba" Página/12. Recuperado de: http://www.pagina12.com.ar/diario/elpais/1-20664-2003-05-27.html .

Bruschtein, L. (24 de abril de 2010). La generación del Bicentenario. Página/12. Recuperado de: http:// www.pagina12.com.ar/diario/elpais/1-144529-2010-04-24.html .

Castro, F. (2009). Latinoamericanismo vs. Imperialismo. México D.F.: Océano Sur.

Ceceña, A. (2008). El posneoliberalismo y sus bifurcaciones. Observatorio Latinoamericano de Geopolítica. Recuperado de: http://www.geopolitica.ws.

CELAC (2011). Declaración de Caracas. Recuperado de: http:// www.juventudrebelde.cu/internacionales/2011-12-04/ declaracion-de-caracas-sobre-celac/.

Chavez, H. (1994). Discurso de Hugo Chávez en Cuba. De: https://www.youtube.com/watch?v=G75tVRv2LQo.

Correo del Orinoco (13 de marzo de 2013). Rememoran primera visita de Chávez a Cuba. Correo del Orinoco. Recuperado de: http://www.correodelorinoco.gob.ve/politica/rememoran-primera-visita-chavez-a-cuba/.

Cuevas, R. (2008). Sandino y la intelectualidad costarricense. Nacionalismo antiimperialista en Nicaragua y Costa Rica (1927-1934). San José:EUNED.

Gamboa, Emma (1971). Omar Dengo. San José, CR: Ministerio de Cultura, Juventud y Deportes. Pp. 214-216.

González Casanova, P. (1991) Imperialismo y liberación. Una introducción a la historia contemporánea de América Latina. México, D.F.: Siglo XXI Editores. 
Kirchner, N. (2005). Palabras del presidente de la república argentina, Dr. Néstor Kirchner durante la inauguración de la IV Cumbre de las Américas, en Mar del Plata. Recuperado de: http:// www.oas.org/es/centro_noticias/discurso.asp?sCodigo $=05-0263$

Martí, J. (1999) Ismaelillo. Versos libres. Versos sencillos. Madrid: Jorge Mestas Ediciones.

Martí, J. (1889). Congreso Internacional de Washington. Su historia, sus elementos y sus tendencias (Carta al director de La Nación de Argentina), New York, 2 de noviembre de 1889. En: Martí, J. (2005). Nuestra América (Antología), Caracas: Fundación Biblioteca Ayacucho.

Martí, J (1891). Nuestra América. En Hart-Dávalos, Armando (Editor) (2000). José Martí y el equilibrio del mundo. México DF: Fondo de Cultura Económica.

Martí, José (1894). El tercer año del Partido Revolucionario Cubano. En Hart-Dávalos, Armando (Editor) (2000). José Martí y el equilibrio del mundo. México DF: Fondo de Cultura Económica.

Martí, J (1894). La verdad sobre los Estados Unidos. En Hart Dávalos, Armando (Editor) (2000). José Martí y el equilibrio del mundo. México DF: Fondo de Cultura Económica.

Minga Informativa (2006, 9 de diciembre). "Presidente electo ecuatoriano Rafael Correa en la Cumbre Social", Movimientos.org. Recuperado de: http://movimientos.org/pt-br/ node/8759.
Quesada, R. (2001). El legado de la guerra hispano-antillano-norteamericana. San José, Costa Rica: EUNED.

Quesada, R. (2012). América Latina 18102010. El legado de los imperios. San José, C.R.: EUNED.

Regueiro, L. (2008) Los TLC en la perspectiva de la acumulación estadounidense. Visiones desde el Mercosur y el ALBA. Buenos Aires: Consejo Latinoamericano de Ciencias Sociales - CLACSO.

Sandino, A. (s.f.). Plan de realización del supremo sueño de Bolívar. [Documento digitalizado]. Recuperado de: http://exwebserv.telesurtv.net/secciones/archivos/ARCH352_539.pdf.

Suárez, L. (2008). "La integración independiente y multidimensional de Nuestra América: una mirada desde lo mejor del pensamiento sociológico", Política Exterior y Soberanía, 3, (2). Pp. 21-26. 